\title{
Parametric excitation of high-frequency electromagnetic waves by the lower-frequency dipole pumping
}

\author{
K. V. Gamayunov \\ Altai State University, 6560099, Barnaul, Russia \\ G. V. Khazanov \\ Space Physics Research Laboratory, University of Michigan, Ann Arbor, Michigan 48105 \\ E. N. Krivorutsky and A. A. Veryaev \\ Altai State University, 6560099, Barnaul, Russia
}

(Received 23 June 1992; accepted 24 September 1992)

\begin{abstract}
The possibility of parametric excitation of high-frequency electromagnetic waves by lowerfrequency dipole pumping is studied. It is shown that the obtained general dispersive equation may be reduced to the Mathieu equation, provided the case of the flux instability is neglected. In the framework of the developed approach, the excitation of magnetohydrodynamic waves and whistler oscillations is examined.
\end{abstract}

\section{INTRODUCTION}

The problems of the generation of modes of high frequency in the presence of lower-frequency oscillations attract the researcher's attention both in the theoretical and experimental aspects. $^{1-3}$ The authors of the abovementioned papers studied the excitation of Langmuir waves. However, to better understand the physical mechanisms of the generation of modes of high frequency in the presence of lower-frequency oscillations it is necessary to study the behavior of nonpotential oscillations as well. Besides, electrodynamic waves allow us to make use of more diverse means while studying them experimentally.

The present paper considers the excitation of electromagnetic modes by the lower-frequency dipole pumping. Unlike in Refs. 1-3, the analysis is made in the approximation of regular phases (see, for instance, Ref. 4). As is shown below, the smallness of the pumping wave frequency and neglect of the flux instability allow us to analyze the dispersive equation of arbitrary electromagnetic oscillations by reducing the task to the solution of the Mathieu equation. However, this paper is limited to concentrating on certain cases of a particular interest.

\section{THE DISPERSIVE EQUATION OF ELECTROMAGNETIC OSCILLATIONS IN THE FIELD OF DIPOLE PUMPING}

The dispersive equation of electromagnetic oscillations in the field of the dipole pumping is known fairly well (see, for instance, Ref. 4 and references therein). However, below we will obtain this equation in a different, more convenient form for us.

Let us consider a homogeneous plasma in the magnetic field $\mathbf{B}_{0}$ oriented along axis $z\left(\mathbf{B}_{0} \| \mathbf{z}\right)$. Let the plasma be acted upon by the dipole harmonic field of pumping $\mathbf{E}_{0}\left(\omega_{0} t\right)$. (Subsequently, we will neglect the magnetic field of the pumping wave everywhere.) Then electrons and ions in the field of the pumping will have speed $\mathrm{U}_{0 \alpha}\left(\omega_{0} t\right)$ and oscillation coordinates $\mathbf{r}_{0 \alpha}\left(\omega_{0} t\right)$ (for the electron-proton plasma $\alpha=i, e$ ).
The dispersive equation of electromagnetic oscillations will be obtained in the coordinate system, connected with macroscopically moving electrons. In this coordinate system, the field of pumping does not act upon the electrons and the linearized kinetic equations for the disturbance of the distribution function $f_{e}$ has a well-known form: ${ }^{5}$

$$
\begin{gathered}
\frac{\partial f s b e}{\partial t}+\mathbf{w} \cdot \frac{\partial f_{e}}{\partial \mathbf{r}}+\omega_{B e}\left(w \times \frac{\mathbf{B}_{0}}{B_{0}}\right) \cdot \frac{\partial f_{e}}{\partial w} \\
=\frac{|e|}{m_{e}}\left(\mathbf{E}+\frac{1}{c}(\boldsymbol{w} \times \mathbf{B})\right) \cdot \frac{\partial f_{0 e}}{\partial \mathbf{w}},
\end{gathered}
$$

where $\omega_{B e}$ is the gyrofrequency, $f_{0 e}$ is the undisturbed electron distribution function, and the rest of the notation is standard.

Going over into the Fourier representation in Eq. (1),

$$
A(\mathbf{r}, t)=\int e^{-i \omega t+i \mathbf{k r}} A(\omega, \mathbf{k}) \frac{d \omega d \mathbf{k}}{(2 \pi)^{4}},
$$

and using the Maxwell equation

$$
\operatorname{rot} \mathbf{E}=-\frac{1}{c} \frac{\partial \mathbf{B}}{\partial t}
$$

we can write the expression for the electron current: ${ }^{5}$

$$
j_{m}^{(e)}=\sigma_{m n}^{(e)} E_{n} .
$$

Here, $\sigma_{m n}^{(\alpha)}$ is the linear conduction of $\alpha$-sort particles that, obviously, has the form similar to the one in the plasma without macroscopic motion. ${ }^{5}$

In order to write the dispersive equation, we will have to calculate the linear, relative to the field of excitation, ion current in the electron coordinate system.

If we go over into the coordinate system connected with macroscopically moving ions, in the linearized kinetic equation for ions, we thereby shall exclude the pumping field. Then, just as for electrons, we can write

$$
\tilde{J}_{m}^{(i)}=\sigma_{m n}^{(i)} \widetilde{E}_{n}
$$


where the tilde indicates that the values are taken in the ion coordinate system and $\sigma_{m n}^{(i)}$ has the form similar to the one in the plasma without the macroscopic motion. Thus, to write the ion current in the electron system of coordinates, we will have to transform Eq. (4) into this system.

In the electron coordinate system, the pumping field causes the movement of ions with the following values of coordinates and velocities: ${ }^{5,6}$

$$
\begin{aligned}
& \mathbf{r}_{0}(t)=\left(\begin{array}{l}
\left(r_{0 i}^{x}-r_{0 e}^{x}\right) \cos \omega_{0} t \equiv x_{0} \cos \omega_{0} t \\
\left(r_{0 i}^{y}-r_{0 e}^{y}\right) \sin \omega_{0} t \equiv y_{0} \sin \omega_{0} t \\
\left(r_{0 i}^{z}-r_{0 e}^{z}\right) \cos \omega_{0} t \equiv z_{0} \cos \omega_{0} t
\end{array}\right), \\
& \mathbf{u}_{0}(t)=\left(\begin{array}{c}
\left(u_{0 i}^{x}-u_{0 e}^{x}\right) \sin \omega_{0} t \equiv u_{1} \sin \omega_{0} t \equiv i V_{1} \sin \omega_{0} t \\
\left(u_{0 i}^{y}-u_{0 e}^{y}\right) \cos \omega_{0} t \equiv u_{2} \cos \omega_{0} t \equiv V_{2} \cos \omega_{0} t \\
\left(u_{0 i}^{z}-u_{0 e}^{z}\right) \sin \omega_{0} t \equiv u_{3} \sin \omega_{0} t \equiv i V_{3} \sin \omega_{0} t
\end{array}\right)
\end{aligned}
$$

Moreover, we can write

$$
\begin{aligned}
& \mathbf{k r}_{0}(t)=a \sin \left(\omega_{0} t+\theta\right), \\
& a^{2}=\left(k_{x} x_{0}+k_{z} z_{0}\right)^{2}+k_{y}^{2} y_{0}^{2}, \\
& \theta=\arctan \left(k_{x} x_{0}+k_{z} z_{0}\right) / k_{y} y_{0} .
\end{aligned}
$$

The expression for the ion current in the electron coordinate system has the form

$$
\begin{aligned}
& \mathbf{j}^{(i)}(\mathbf{r}, t)=|e| \int \mathbf{w} f_{i}(\mathbf{r}, t, \mathbf{w}) d \mathbf{w}, \\
& \mathbf{r}=\mathbf{r}_{0}+\mathbf{r}^{\prime}, \quad \mathbf{w}=\mathbf{u}_{0}+\mathbf{w}^{\prime} .
\end{aligned}
$$

Here, the prime indicates that the coordinates and velocities are taken with respect to the ion system of coordinates. Let us go over into the Fourier representation in Eq. (8):

$$
\begin{aligned}
\mathbf{j}^{(i)}(\omega, \mathbf{k})= & \int d t d \mathbf{r}^{\prime} e^{i \omega t-i \mathbf{k r}-i \mathbf{k} \mathbf{r}_{0}(t)}\left[\mathbf{u}_{0}(t) \tilde{\rho}^{(i)}\left(\mathbf{r}^{\prime}, t\right)\right. \\
& \left.+\widetilde{\mathbf{j}}^{(i)}\left(\mathbf{r}^{\prime}, t\right)\right] .
\end{aligned}
$$

After substituting Eqs. (6) and (7) here, and taking into account the continuity equation in the ion coordinate system

$$
\tilde{\rho}^{(i)}(\omega, \mathbf{k})=\mathbf{k} \widetilde{\mathbf{j}}^{(i)}(\omega, \mathbf{k}) / \omega,
$$

we obtain

$$
\begin{aligned}
j_{m}^{(i)}(\omega, \mathbf{k})= & \Delta^{-1}(\mathbf{k})\left[\delta_{m n} \omega+\left(V_{m} k_{n} / 2\right)\left(L^{+1}+G^{m} L^{-1}\right)\right] \\
& \times\left[\tilde{j}_{n}^{(i)}(\omega, \mathbf{k}) / \omega\right] \\
& m, n=1,2,3 \equiv x, y, z .
\end{aligned}
$$

Here, we introduce the operators

$$
\begin{gathered}
A(\omega, \mathbf{k})=\sum_{n} e^{-i n \theta} J_{n}(a) \tilde{A}\left(\omega-n \omega_{0}, \mathbf{k}\right) \\
\equiv \Delta^{-1}(\mathbf{k}) \tilde{A}(\omega, \mathbf{k}) \\
\tilde{A}\left(\omega \pm \omega_{0}, \mathbf{k}\right)=L^{ \pm 1} \tilde{A}(\omega, \mathbf{k})
\end{gathered}
$$

where $\delta_{m n}$ is the Kronecker delta and $G^{m} \equiv(-1)^{m}$. Using the transformation for the field ${ }^{7}$

$$
\widetilde{\mathbf{E}}\left(\mathbf{r}^{\prime}, t\right)=\mathbf{E}(\mathbf{r}, t)+(1 / c)\left[\mathbf{u}_{0}(t) \times \mathbf{B}(\mathbf{r}, t)\right]
$$

and the Maxwell equation (2) in the electron coordinate system, we obtain

$$
\begin{aligned}
\tilde{E}_{i}(\omega, \mathbf{k})= & \Delta(\mathbf{k})\left[\delta_{i j}\left(\omega-\frac{k_{n} V_{n}}{2}\left(L^{+1}+G^{n} L^{-1}\right)\right)\right. \\
& \left.+\frac{V_{j} k_{i}}{2}\left(L^{+1}+G^{j} L^{-1}\right)\right] \frac{E_{j}(\omega, \mathbf{k})}{\omega} .
\end{aligned}
$$

Here,

$$
\widetilde{A}(\omega, \mathbf{k})=\sum_{n} e^{i n \theta} J_{n}(\alpha) A\left(\omega+n \omega_{0}, \mathbf{k}\right) \equiv \Delta(\mathbf{k}) A(\omega, \mathbf{k}),
$$

and the combination $a_{i} b_{i} G^{i}$ is written as follows:

$$
a_{i} b_{i} G^{i}=-a_{1} b_{1}+a_{2} b_{2}-a_{3} b_{3} .
$$

From Eqs. (10), (4), and (11) we obtain the final expression for the ion current in the electron coordinate system:

$$
\begin{aligned}
j_{k}^{(i)}(\omega, \mathbf{k})= & \Delta^{-1}(\mathbf{k})\left(\delta_{k e} \omega+\frac{V_{k} k_{e}}{2}\left(L^{+1}+G^{k} L^{-1}\right)\right) \\
& \times \frac{\sigma_{e m}^{(i)}(\omega, \mathbf{k})}{\omega} \Delta(\mathbf{k}) \\
& \times\left[\delta_{m n}\left(\omega-\frac{V_{s} k_{s}}{2}\left(L^{+1}+G^{s} L^{-1}\right)\right)\right. \\
& \left.+\frac{V_{n} k_{m}}{2}\left(L^{+1}+G^{n} L^{-1}\right)\right] \frac{E_{n}(\omega, \mathbf{k})}{\omega},
\end{aligned}
$$

or in an expanded form:

$$
\begin{aligned}
j_{k}^{(i)}(\omega, \mathbf{k})= & \sum_{p, q} e^{i q \theta} I_{p}(a) J_{p+q}(a)\left(\delta_{k e}\left(\omega-p \omega_{0}\right)+\frac{V_{k} k_{e}}{2}\left(L^{+1}+G^{k} L^{-1}\right)\right) \frac{\sigma_{e m}^{(i)}\left(\omega-p \omega_{0}, \mathbf{k}\right)}{\omega-p \omega_{0}} \\
& \times\left[\delta_{m n}\left(\left(\omega+q \omega_{0}\right)-\frac{V_{s} k_{s}}{2}\left(L^{+1}+G^{s} L^{-1}\right)\right)+\frac{V_{n} k_{m}}{2}\left(L^{+1}+G^{n} L^{-1}\right)\right] \frac{E_{n}\left(\omega+q \omega_{0}, \mathbf{k}\right)}{\omega+q \omega_{0}}
\end{aligned}
$$


The Maxwell equations (2) and

$$
\operatorname{rot} \mathbf{B}=\frac{4 \pi}{c} \mathbf{j}+\frac{1}{c} \frac{\partial \mathbf{E}}{\partial t},
$$

using Eqs. (3) and (12), allow us to write the dispersive equation:

$$
\begin{aligned}
& \left(\frac{c^{2}}{\omega^{2}}\left(k_{i} k_{j}-\delta_{i j} k^{2}\right)+\delta_{i j}+\frac{4 \pi i}{\omega} \sigma_{i j}^{(e)}\right) E_{j}(\omega, \mathbf{k}) \\
& +\frac{4 \pi i}{\omega} j_{i}^{(i)}(\omega, \mathbf{k})=0 .
\end{aligned}
$$

The dispersive equation (14) describes the parametric interaction of arbitrary oscillations with the dipole pumping wave.

Let us simplify Eq. (14) with reference to our case, namely, to the excitation of high-frequency modes in the presence of the lower-frequency pumping wave, Expanding the electric field and conduction into the Taylor series with respect to point $(\omega, \mathbf{k})$ in Eq. (12b), we can sum over $p$ and $q$. Next, using $\omega_{0}<\omega$ and $k u_{0} \alpha<\omega$ (the fulfillment of the latter inequality is necessary in order to exclude the flux instability), we can restrict ourselves to terms $t=0,1,2$ in the conduction expansion (see Appendix A). Retaining the terms proportional to $1, k u_{0}, k u_{0} \omega_{0},\left(k u_{0}\right)^{2}$ and convolving the expansion of the electric field, we finally obtain:

$$
j_{k}^{(i)}(\omega, \mathbf{k})=\sigma_{k e}^{(i)}(\omega) E_{e}(\omega)+J_{k}(\omega, \mathbf{k}),
$$

where $J_{k}(\omega, \mathbf{k})$ is the part of the ion current dependent on the pumping field. A detailed derivation of Eq. (15) and the expression for $J_{k}(\omega, \mathbf{k})$ are given in Appendix A.

Using Eq. (15), the dispersive equation (14) can be written as

$$
\begin{gathered}
{\left[\left(c^{2} / \omega^{2}\right)\left(k_{i} k_{j}-\delta_{i j} k^{2}\right)+\delta_{i j}+\chi_{i j}\right] E_{j}(\omega, \mathbf{k})} \\
+(4 \pi i / \omega) J_{i}(\omega, \mathbf{k})=0
\end{gathered}
$$

where

$$
\chi_{i j}=\chi_{i j}^{(e)}+\chi_{i j}^{(i)},
$$

$\chi_{i j}^{(\alpha)}$ is the linear susceptibility of $\alpha$-sort particles. The analysis of the dispersive equation (16) in a general form is rather complicated, therefore, in the paragraphs below, we shall consider the most characteristic, in our view, particular cases. Besides, it will be clear from the consideration of these particular cases, how to analyze the parametric interaction of arbitrary modes with the pumping wave.

\section{EXCITATION OF MHD WAVES PROPAGATING ALONG THE EXTERNAL MAGNETIC FIELD BY THE PUMPING FIELD $E_{0} \perp B_{0}$}

Let us introduce the polarization vector for magnetohydrodynamic (MHD) waves with the wave vector $\mathbf{k}$ along the external magnetic field: ${ }^{5}$

$$
\mathbf{e}(\omega, \mathbf{k})=\left[e_{1}(\omega, \mathbf{k}), e_{2}(\omega, \mathbf{k})\right] \equiv\left[e_{1}(\omega, \mathbf{k}), \tilde{i e_{2}}(\omega, \mathbf{k})\right] .
$$

Then, $J_{k}(\omega, \mathbf{k})$ assumes the form [for brevity's sake we will use $G^{n}$ instead of $G_{n k}$ and $\left.E_{e}\left(\omega \pm n \omega_{0}\right) \equiv E_{e}^{ \pm n}\right]$ :

$$
\begin{aligned}
J_{k}(\omega, \mathbf{k})= & \frac{V_{k} k^{2} \sigma_{33}^{(i)}}{4 \omega^{2}}\left\langle\omega V_{e}\left(\frac{E_{e}^{+2}}{\omega+2 \omega_{0}}+G^{k} G^{e} \frac{E_{e}^{-2}}{\omega-2 \omega_{0}}\right)\right. \\
& \left.+V_{e} E_{e}(\omega)\left(G^{e}+G^{k}\right)\right\rangle .
\end{aligned}
$$

Writing Eq. (18), we have made use of the fact that $\sigma_{13}=\sigma_{23}=0$. The smallness of the pumping frequency $\omega_{0}<\omega$ allows us to simplify

$$
E_{k}\left(\omega \pm n \omega_{0}\right) \simeq e_{k}(\omega) E\left(\omega \pm n \omega_{0}\right),
$$

then Eq. (18) assumes the form

$$
\begin{aligned}
J_{k}(\omega, \mathrm{k})= & \frac{V_{k} k^{2} \sigma_{33}^{(i)}}{4 \omega^{2}}\left\langle\omega \left(\frac{V_{1} e_{1}+i V_{2} \widetilde{e}_{2}}{\omega+2 \omega_{0}} E^{+2}\right.\right. \\
& \left.+G^{k} \frac{i V_{2} \widetilde{e}_{2}-V_{1} e_{1}}{\omega-2 \omega_{0}} E^{-2}\right)+\left[i V_{2} \widetilde{e}_{2}-V_{1} e_{1}\right. \\
& \left.\left.+G^{k}\left(V_{1} e_{1}+i V_{2} \widetilde{e}_{2}\right)\right] E(\omega)\right\rangle
\end{aligned}
$$

Substituting the current equation (20) into Eq. (16), convolving with the components of the polarization vector $e_{i}^{*}(\omega)$, and, taking into account Eq. (6), we obtain the dispersive equation

$$
\begin{aligned}
\Lambda(\omega) E(\omega)= & \frac{k^{2} \chi_{33}^{(i)}}{4 \omega^{2}}\left\langle\left(u_{1}^{2} e_{1}^{2}-u_{2}^{2} \vec{e}_{2}^{2}\right)\left(E^{+2}+E^{-2}\right)\right. \\
& \left.-2\left(u_{1}^{2} e_{1}^{2}+u_{2}^{2} \vec{e}_{2}^{2}\right) E(\omega)\right\rangle .
\end{aligned}
$$

Writing Eq. (21), we omitted the terms proportional to $\omega_{0} / \omega$ and introduced the notation

$$
\Lambda(\omega)=1+e_{i}^{*} \chi_{i j} f_{j}-\frac{c^{2} k^{2}}{\omega^{2}} .
$$

To solve Eq. (21), it is necessary to know the polarization vector. Taking into account the smallness of $k u_{0} \alpha / \omega$, let us assume that the functional dependence of the polarization vector on $(\omega, \mathbf{k})$ and the medium parameters remain the same as in the plasma without the pumping field. Note, also, that since $\omega_{0}<\omega$, then to solve the dispersive equation, it is more convenient to go over into the $t$ space. Before going over into the $t$ space in Eq. (21), let us carry out obvious simplifications.

The inequality $k u_{0} \alpha<\omega$ allows us in Eq. (21) to expand into the series with respect to the solution of the undisturbed dispersive equation:

$$
\Lambda\left(\omega_{\mathbf{k}}\right)=0, \quad \omega_{\mathbf{k}}=\omega_{*}+i \delta,
$$

where $\omega_{*}$ is close to $\omega$. Besides, assuming $\delta$ to be a small value, we will take into account the imaginary part of susceptibilities only in the term of the zero approximation, i.e., in Eq. (22) and further expand with respect to $\omega_{*}$. Then, Eq. (21) takes the form 


$$
\begin{aligned}
& \left\langle[\Lambda]_{*}^{\prime}\left(\omega-\omega_{*}\right)+\frac{1}{2}[\Lambda]_{*}^{\prime \prime}\left(\omega-\omega_{*}\right)^{2}\right\rangle E(\omega) \\
& =\frac{k^{2}}{4}\left[( E ^ { + 2 } + E ^ { - 2 } ) \left\langle\left(\frac{\chi_{33}^{(i)}}{\omega^{2}}\left(u_{1}^{2} e_{1}^{2}-u_{2}^{2} \vec{e}_{2}^{2}\right)\right)_{*}\right.\right. \\
& \left.+\left(\frac{\chi_{33}^{(i)}}{\omega^{2}}\left(u_{1}^{2} e_{1}^{2}-u_{2}^{2} e_{2}^{2}\right)\right)_{*}^{\prime}\left(\omega-\omega_{*}\right)\right\rangle \\
& -2 E(\omega)\left\langle\left(\frac{\chi_{33}^{(i)}}{\omega^{2}}\left(u_{1}^{2} e_{1}^{2}+u_{2}^{2} \vec{e}_{2}^{2}\right)\right)_{*}\right. \\
& \left.\left.+\left(\frac{\chi_{33}^{(i)}}{\omega^{2}}\left(u_{1}^{2} e_{1}^{2}+u_{2}^{2} \vec{e}_{2}^{2}\right)\right)_{*}^{\prime}\left(\omega-\omega_{*}\right)\right\rangle\right],
\end{aligned}
$$

where $[\cdots]_{*}^{\prime}$ and $[\cdots]_{*}^{\prime \prime}$ are the first and second derivatives over $\omega$ calculated at point $\omega_{*}$, and $(\cdots)_{*}$ is the function value at this point. Going over in Eq. (23) to the $t$ representation

$$
X(t)=\int e^{-i \omega t} X(\omega) \frac{d \omega}{2 \pi},
$$

we obtain

$$
\begin{gathered}
\frac{\partial^{2} E(t, \mathbf{k})}{\partial t^{2}}+\left(A+B \cos 2 \omega_{0} t\right) \frac{\partial E(t, \mathbf{k})}{\partial t} \\
+\left(C+\mathscr{D} \cos 2 \omega_{0} t\right) E(t, \mathbf{k})=0 .
\end{gathered}
$$

Writing Eq. (25), we again used the smallness of $\omega_{0} / \omega_{*}$ and introduced the following notation:

$$
\begin{aligned}
A(\mathbf{k})= & -\frac{2 i}{\Lambda^{\prime \prime}\left(\omega_{*}, \mathbf{k}\right)}\left\langle\Lambda^{\prime}\left(\omega_{*}, \mathbf{k}\right)-\omega_{*} \Lambda^{\prime \prime}\left(\omega_{*}, \mathbf{k}\right)\right. \\
& \left.+\frac{k^{2}}{2}\left(\frac{\chi_{33}^{(i)}}{\omega^{2}}\left(u_{1}^{2} e_{1}^{2}+u_{2}^{2} e_{2}^{2}\right)\right)_{*}^{\prime}\right\rangle \\
B(\mathbf{k})= & {\left[i k^{2} / \Lambda^{\prime \prime}\left(\omega_{*}, \mathbf{k}\right)\right]\left[\left(\chi_{33}^{(i)} / \omega^{2}\right)\left(u_{1}^{2} e_{1}^{2}-u_{2}^{2} \vec{e}_{2}^{2}\right)\right]_{*}^{\prime} } \\
C(\mathbf{k})= & \frac{2}{\Lambda^{\prime \prime}\left(\omega_{*}, \mathbf{k}\right)}\left[\omega_{*} \Lambda^{\prime}\left(\omega_{*}, \mathbf{k}\right)-\frac{\omega_{*}^{2}}{2} \Lambda^{\prime \prime}\left(\omega_{*}, \mathbf{k}\right)\right. \\
& -\frac{k^{2}}{2}\left\langle\left(\frac{\chi_{33}^{(i)}}{\omega^{2}}\left(u_{1}^{2} e_{1}^{2}+u_{2}^{2} \vec{e}_{2}^{2}\right)\right)_{*}\right. \\
& \left.\left.-\omega_{*}\left(\frac{\chi_{33}^{(i)}}{\omega^{2}}\left(u_{1}^{2} e_{1}^{2}+u_{2}^{2} \vec{e}_{2}^{2}\right)\right)_{*}^{\prime}\right\rangle\right] \\
\mathscr{D}(\mathbf{k})= & \frac{k^{2}}{\Lambda^{\prime \prime}\left(\omega_{*}, \mathbf{k}\right)}\left\langle\left(\frac{\chi_{33}^{(i)}}{\omega^{2}}\left(u_{1}^{2} e_{1}^{2}-u_{2}^{2} \vec{e}_{2}^{2}\right)\right)_{*}\right. \\
& \left.-\omega_{*}\left(\frac{\chi_{33}^{(i)}}{\omega^{2}}\left(u_{1}^{2} e_{1}^{2}-u_{2}^{2} \vec{e}_{2}^{2}\right)\right)_{*}^{\prime}\right\rangle .
\end{aligned}
$$

Substituting $^{8}$

$$
E(t)=\bar{E}(t) e^{-(1 / 2)\left[A t+\left(B / 2 \omega_{0}\right) \sin 2 \omega_{0} t\right]}
$$

reduces Eq. (25) to the Mathieu equation:

$$
\begin{aligned}
& \frac{\partial^{2} \bar{E}(t)}{\partial t^{2}}+\left[C-\frac{1}{4}\left(A^{2}+\frac{B^{2}}{2}\right)\right. \\
& \left.+\left(\mathscr{D}-\frac{A B}{2}\right) \cos 2 \omega_{0} t\right] \bar{E}(t)=0 .
\end{aligned}
$$

The solution of the Mathieu equation within the framework employed while obtaining it is given in Appendix B. To make use of the results of Appendix B, it is necessary, giving the form of susceptibilities and vector of polarization, to calculate the coefficients of Eq. (27).

Let us assume that electrons and ions of the plasma are cold. Then, ${ }^{5}$

$$
\begin{aligned}
& \chi_{i j}=\left(\begin{array}{cc}
\chi_{1} & i \chi_{2} \\
-i \chi_{2} & \chi_{1}
\end{array}\right), \quad \chi_{1}=-\sum_{\alpha} \frac{\omega_{p \alpha}^{2}}{\omega^{2}-\omega_{B \alpha}^{2}}, \\
& \chi_{2}=-\sum_{\alpha} \frac{\omega_{p \alpha}^{2} \omega_{B \alpha}}{\omega\left(\omega^{2}-\omega_{B \alpha}^{2}\right)},
\end{aligned}
$$

where $\omega_{p \alpha}$ is the plasma frequency of $\alpha$-sort particles. Expression $\chi_{33}^{(i)}$ has the form

$$
\chi_{33}^{(i)}=-\omega_{p i}^{2} / \omega^{2},
$$

and for the components of the polarization vector, we can write ${ }^{5}$

$$
\begin{aligned}
& e_{1}=\frac{1}{\left[1+\chi_{2}^{2} /\left(N^{2}-1-\chi_{1}\right)^{2}\right]^{1 / 2}}, \\
& \widetilde{e}_{2}=-\frac{\chi_{2} /\left(N^{2}-1-\chi_{1}\right)}{\left[1+\chi_{2}^{2} /\left(N^{2}-1-\chi_{1}\right)^{2}\right]^{1 / 2}} \\
& N^{2}=\frac{c^{2} k^{2}}{\omega^{2}}
\end{aligned}
$$

The solution of Eq. (22) yields 9

$$
N^{2}-1-\chi_{1}=\xi \chi_{2},
$$

where $\xi=I$ for the left-hand-polarized $(A-)$ and $\xi=-I$ for the right-hand-polarized fast magnetosonic (FMS) waves. In the case of low-frequency $\left(\omega_{*}<\omega_{B i}\right)$ MHD oscillations, the form of susceptibility is considerably simplified:

$$
\chi_{1}=\omega_{p i}^{2} / \omega_{B i}^{2}, \quad \chi_{2}=\omega_{p i}^{2} \omega / \omega_{B i}^{3}
$$

and $\omega_{*}^{2} \simeq k^{2} v_{A}^{2}$ for both types of oscillations $\left(v_{A}^{2} / C^{2}=\omega_{B i}^{2} / \omega_{p i}^{2}\right)$.

Before giving the expressions for the coefficients of Eq. (27), the following remark should be made. Since $\chi_{2}<\chi_{1}$, then while calculating derivatives over $\omega$ from the components of the polarization vector, a large parameter $\omega_{B i} / \hat{\omega}_{*}$ will appear. Therefore, while calculating the coefficients of the Mathieu equation from the components of the polarization vector we will take the derivatives of the first order. 
Omitting simple, but rather unwieldy, calculations, we will write the final form of the coefficients in Eqs. (26) and (27):

$$
\begin{aligned}
& A=\frac{i}{3}\left(8 \omega_{*}+\xi \frac{k^{2} \omega_{B i}^{3}}{2 \omega_{*}^{4}}\left(u_{1}^{2}-u_{2}^{2}\right)\right), \\
& B=-\xi i\left(k^{2} \omega_{B i}^{3} / 6 \omega_{*}^{4}\right)\left(u_{1}^{2}+u_{2}^{2}\right), \\
& C-\frac{1}{4}\left(A^{2}+\frac{B^{2}}{2}\right)=\frac{\omega_{*}^{2}}{9}+\frac{k^{2} \omega_{B i}^{2}}{6 \omega_{*}^{2}}\left(\xi \frac{\omega_{B i}}{3 \omega_{*}}\left(u_{1}^{2}-u_{2}^{2}\right)\right. \\
& -\frac{1}{2}\left(u_{1}^{2}+u_{2}^{2}\right)+\frac{k^{2} \omega_{B i}^{4}}{48 \omega_{*}^{6}} \\
& \left.\times\left(3 u_{1}^{4}-2 u_{1}^{2} u_{2}^{2}+3 u_{2}^{4}\right)\right), \\
& \mathscr{D}-\frac{A B}{2}=-\xi \frac{k^{2} \omega_{B i}^{3}}{18 \omega_{*}^{3}}\left(u_{1}^{2}+u_{2}^{2}\right) .
\end{aligned}
$$

In the expression for $C-\frac{1}{4}\left(A^{2}+B^{2} / 2\right)$, we have written out the two last terms because, as will be seen below, during the calculation of the nonlinear frequency shift, the terms of a higher order are compensated for. From comparing Eqs. (27) and (B1) it is easy to obtain expressions for $h$ and $\theta$ :

$$
\begin{aligned}
& h=\frac{1}{\omega_{0}^{2}}\left[C-\frac{1}{4}\left(A^{2}+\frac{B^{2}}{2}\right)\right], \quad 2 \theta=-\frac{1}{\omega_{0}^{2}}\left(\mathscr{D}-\frac{A B}{2}\right), \\
& z=\omega_{0} t .
\end{aligned}
$$

Now we can use the results obtained in Appendix B (naturally, we assume that all restrictions for $h$ and $\theta$, cited in Appendix B, are met).
Expression (B12), using Eq. (32), yields the growth rate of MHD oscillations:

$$
\frac{\gamma}{\omega_{*}}=\frac{9}{32 \sqrt{2}}\left[\frac{k^{2}\left(u_{1}^{2}+u_{2}^{2}\right)}{\omega_{*}^{2}}\left(\frac{\omega_{B i}}{\omega_{*}}\right)^{3}\right]^{2}\left(\frac{\omega_{0}}{\omega_{*}}\right)^{3} .
$$

To obtain the MHD oscillations' frequency in the presence of the pumping wave, we must use Eqs. (B9), (B11), (32) and the expression for the coefficient $A$ [see Eq. (26)]. After simple calculations, we will obtain

$$
\begin{aligned}
\omega= & \omega_{*}+\left(k^{2} \omega_{B i}^{2} / 8 \omega_{*}^{3}\right)\left[\left(u_{1}^{2}+u_{2}^{2}\right)-\left(k^{2} \omega_{B i}^{4} / 12 \omega_{*}^{6}\right)\right. \\
& \left.\times\left(u_{1}^{2}-u_{2}^{2}\right)^{2}\right] \equiv \omega_{*}+\Delta \omega .
\end{aligned}
$$

It is seen that the last term in Eq. (34) is substantial with sufficiently small $\omega_{*}$ and, besides, it disappears in the case of circular pumping polarization.

In conclusion of this section, we will give the expression for the polarization vector of MHD waves. Substituting the obtained frequency value into Eq. (29), and retaining the first term of the expansion, we obtain

$$
\mathbf{e}=\frac{1}{\sqrt{2}}\left\langle\left(1-\xi \frac{\omega_{B i} \Delta \omega}{\omega_{*}^{2}}\right),-i\left(\xi+\frac{\omega_{B i} \Delta \omega}{\omega_{*}^{2}}\right)\right) .
$$

The pumping field transforms the polarization of MHD waves from the circular into the elliptic one, and the large semiaxes of $A$ and FMS waves ellipses are mutually perpendicular.

\section{EXCITATION OF MHD WAVES, PROPAGATING ALONG THE EXTERNAL MAGNETIC FIELD, BY THE PUMPING FIELD $E_{0} \| B_{0}$}

While considering MHD waves with $\mathbf{k} \| \mathbf{B}_{0}$ in the pumping field $\mathbf{E}_{0}$ il $\mathbf{B}_{0}$ the expression for current $J_{k}(\omega, \mathbf{k})$ assumes the form [see Eq. (A5)]

$$
\begin{aligned}
J_{k}(\omega, \mathbf{k})= & -\frac{a \omega_{0}}{2} \frac{\partial \sigma_{k e}^{(i)}}{\partial \omega}\left(E_{e}^{+1} e^{i \theta}+E_{e}^{-1} e^{-i \theta}\right)-\frac{a \omega_{0}^{2}}{4} \frac{\partial^{2} \sigma_{k e}^{(i)}}{\partial \omega^{2}}\left\langle E_{e}^{+1} e^{i \theta}-E_{e}^{-1} e^{-i \theta}-\frac{a}{2}\left[E_{e}^{+2} e^{i 2 \theta}+E_{e}^{-2} e^{-i 2 \theta}+2 E_{e}(\omega)\right]\right\rangle \\
& -\frac{k V_{3}}{2}\left\langle\sigma_{k e}^{(i)}\left(\frac{E_{e}^{+1}}{\omega+\omega_{0}}-\frac{E_{e}^{-1}}{\omega-\omega_{0}}\right)-\frac{a \omega_{0}}{2} \frac{\partial \sigma_{k e}^{(i)}}{\partial \omega}\left(e^{i \theta} \frac{E_{e}^{+2}}{\omega+2 \omega_{0}}-e^{-i \theta} \frac{E_{e}^{-2}}{\omega-2 \omega_{0}}+\frac{E_{e}(\omega)}{\omega}\left(e^{-i \theta}-e^{i \theta}\right)\right)\right\rangle .
\end{aligned}
$$

Using Eq. (19), we obtain the dispersive equation

$$
\begin{gathered}
\Lambda(\omega) E(\omega)-\frac{a \omega_{0}}{2 \omega} e_{m}^{*} \frac{\partial}{\partial \omega}\left(\omega \chi_{m n}^{(i)}\right) e_{n}\left(e^{i \theta} E^{+1}+e^{-i \theta} E^{-1}\right) \\
+\frac{a^{2} \omega_{0}^{2}}{4 \omega^{2}} e_{m}^{*} \frac{\partial}{\partial \omega}\left(\omega^{2} \frac{\partial \chi_{m n}^{(i)}}{\partial \omega}\right) e_{n} E(\omega)+\operatorname{sig}\left(k z_{0}\right) i \frac{a \omega_{0}}{2} \\
\quad \times\left\langle\frac{1}{\omega} e_{m}^{*} \chi_{m n}^{(i)} e_{n}\left(E^{+1}-E^{-1}\right)+\operatorname{sig}\left(k z_{0}\right) i \frac{a \omega_{0}}{\omega^{2}} e_{m}^{*} \frac{\partial}{\partial \omega}\right.
\end{gathered}
$$

$$
\left.\times\left(\omega \chi_{m n}^{(i)}\right) e_{n} E(\omega)\right\rangle=0 .
$$

While obtaining Eq. (37), we neglected the terms that are proportional to $\omega_{0} / \omega$ and $\left[\left(a \omega_{0}\right)^{2} / \omega^{2}\right]\left(E^{+2} \pm E^{-2}\right)$, and used the obvious relationships [sec Eqs. (6) and (7)]:

$$
k u_{3}=-a \omega_{0} \operatorname{sig}\left(k z_{0}\right), \quad \theta=(\pi / 2) \operatorname{sig}\left(k z_{0}\right) .
$$

Expanding into the series with respect to the undisturbed solution, the dispersive equation (37) assumes the form 


$$
\begin{aligned}
& \left\langle[\Lambda]_{*}^{\prime}\left(\omega-\omega_{*}\right)+\frac{1}{2}[\Lambda]_{*}^{\prime \prime}\left(\omega-\omega_{*}\right)^{2}\right\rangle E(\omega)-\frac{a \omega_{0}}{2}\left(e^{i \theta} E^{+1}+e^{-i \theta} E^{-1}\right)\left\langle\left(\frac{1}{\omega} e_{m}^{*} \frac{\partial}{\partial \omega}\left(\omega \chi_{m n}^{(i)}\right) e_{n}\right)_{*}+\left(\frac{1}{\omega} e_{m}^{*} \frac{\partial}{\partial \omega}\left(\omega \chi_{m n}^{(i)}\right) e_{n}\right)_{*}^{\prime}\right. \\
& \left.\times\left(\omega-\omega_{*}\right)\right\rangle+\frac{a^{2} \omega_{0}^{2}}{4}\left[\frac{e_{m}^{*}}{\omega^{2}} \frac{\partial}{\partial \omega}\left(\omega^{2} \frac{\partial \chi_{m n}^{(i)}}{\partial \omega}\right) e_{n}\right]_{*} E(\omega)-\operatorname{sig}\left(k z_{0}\right) i \frac{a \omega_{0}}{2}\left[( E ^ { + 1 } - E ^ { - 1 } ) \left(\left(\frac{1}{\omega} e_{m}^{*} \chi_{m n}^{(i)} e_{n}\right)_{*}+\left(\frac{1}{\omega} e_{m}^{*} \chi_{m n}^{(i)} e_{n}\right)_{*}^{\prime}\right.\right. \\
& \left.\left.\quad \times\left(\omega-\omega_{*}\right)\right\rangle+\operatorname{sig}\left(k z_{0}\right) i a \omega_{0}\left(\frac{e_{m}^{*}}{\omega^{2}} \frac{\partial}{\partial \omega}\left(\omega \chi_{m n}^{(i)}\right) e_{n}\right)_{*} E(\omega)\right]=0
\end{aligned}
$$

To go over into the $t$ space, it is more convenient to use the Fourier transformation with a shifted zero time reference, rather than Eq. (24):

$$
X(\tau)=\int e^{-i \omega\left(\tau+\pi / 2 \omega_{0}\right)} X(\omega) \frac{d \omega}{2 \pi},
$$

After simple transformations, Eq. (38) will have the form

$$
\begin{gathered}
\frac{\partial^{2} E(\tau, \mathbf{k})}{\partial \tau^{2}}+\left(A+B \cos \omega_{0} \tau\right) \frac{\partial E(\tau, \mathbf{k})}{\partial \tau} \\
+\left(C+\mathscr{D} \cos \omega_{0} \tau\right) E(\tau, \mathbf{k})=0 .
\end{gathered}
$$

Here, as before, we have taken into account the smallness of $\omega_{0} / \omega_{*}$ and introduced the notation

$$
\begin{aligned}
& A(\mathbf{k})=-\left[2 i / \Lambda^{\prime \prime}\left(\omega_{*}, \mathrm{k}\right)\right]\left\langle\Lambda^{\prime}\left(\omega_{*}, \mathrm{k}\right)-\omega_{*} \Lambda^{\prime \prime}\left(\omega_{*}, \mathrm{k}\right)\right\rangle \text {, } \\
& B(\mathbf{k})=-\frac{2 i a \omega_{0}}{\Lambda^{\prime \prime}\left(\omega_{*}, \mathbf{k}\right)} \operatorname{sig}\left(k z_{0}\right) \cdot\left(\left(\frac{1}{\omega} e_{m}^{*} \chi_{m n}^{(i)} e_{n}\right)_{*}^{\prime}\right. \\
& \left.+\left(\frac{1}{\omega} e_{m}^{*} \frac{\partial}{\partial \omega}\left(\omega \chi_{m n}^{(i)}\right) e_{n}\right)_{*}^{\prime}\right) \text {. } \\
& C(\mathbf{k})=\frac{2}{\Lambda^{\prime \prime}\left(\omega_{*}, \mathbf{k}\right)}\left\{\omega_{*} \Lambda^{\prime}\left(\omega_{*}, \mathbf{k}\right)-\frac{\omega_{*}^{2}}{2} \Lambda^{\prime \prime}\left(\omega_{*}, \mathbf{k}\right)\right. \\
& -\frac{a^{2} \omega_{0}^{2}}{4}\left\langle\left[\frac{e_{m}^{*}}{\omega^{2}} \frac{\partial}{\partial \omega}\left(\omega^{2} \frac{\partial \chi_{m n}^{(i)}}{\partial \omega}\right) e_{n}\right]_{*}\right. \\
& \left.\left.+2\left(\frac{e_{m}^{*}}{\omega^{2}} \frac{\partial}{\partial \omega}\left(\omega \chi_{m n}^{(i)}\right) e_{n}\right)_{*}\right)\right\} \\
& \mathscr{D}(\mathbf{k})=-\frac{2 a \omega_{0}}{\Lambda^{\prime \prime}\left(\omega_{*}, \mathbf{k}\right)} \operatorname{sig}\left(k z_{0}\right)\left\langle\left(\frac{1}{\omega} e_{m}^{*} \chi_{m n}^{(i)} e_{n}\right)_{*}\right. \\
& -\omega_{*}\left(\frac{1}{\omega} e_{m}^{*} \chi_{m n}^{(i)} e_{n}\right)_{*}^{\prime} \\
& +\left(\frac{1}{\omega} e_{m}^{*} \frac{\partial}{\partial \omega}\left(\omega \chi_{m n}^{(i)}\right) e_{n}\right)_{*} \\
& \left.-\omega_{*}\left(\frac{1}{\omega} e_{m}^{*} \frac{\partial}{\partial \omega}\left(\omega \chi_{m n}^{(i)}\right) e_{n}\right)_{*}^{\prime}\right\rangle .
\end{aligned}
$$

Substituting

$$
E(\tau)=\bar{E}(\tau) e^{-(1 / 2)\left[A \tau+\left(B / \omega_{0}\right) \sin \omega_{0} \tau\right]}
$$

reduces Eq. (40) to the Mathieu equation:

$$
\begin{aligned}
& \frac{\partial^{2} \bar{E}(\tau)}{\partial \tau^{2}}+\left[C-\frac{1}{4}\left(A^{2}+\frac{B^{2}}{2}\right)\right. \\
& \left.\quad+\left(\mathscr{D}-\frac{A B}{2}\right) \cos \omega_{0} \tau\right] \bar{E}(\tau)=0
\end{aligned}
$$

where, just as in obtaining Eq. (27), the terms proportional to $B \omega_{0} \sin \omega_{0} \tau$ and $B^{2} \cos 2 \omega_{0} \tau$, are not taken into account.

Let us consider electrons and ions of the plasma to be cold. Using Eqs. (28)-(31) and omitting simple calculations, let us write the final form of the coefficients in Eq. (41) and the Mathieu equation:

$$
\begin{gathered}
A=i \frac{8}{3} \omega_{*}, \quad B=-\xi \operatorname{sig}\left(k z_{0}\right) \frac{2}{3} i a \omega_{0} \frac{\omega_{B i}}{\omega_{*}}, \\
C-\frac{1}{4}\left(A^{2}+\frac{B^{2}}{2}\right)=\frac{\omega_{*}^{2}}{9}+\frac{a^{2} \omega_{0}^{2} \omega_{B i}^{2 *}}{18 \omega_{*}^{2}}, \\
\ddots \cdots \\
\mathscr{D}-A B / 2=\xi \operatorname{sig}\left(k z_{0}\right) a \omega_{0} \omega_{B i \vartheta}^{5} .
\end{gathered}
$$

From Eqs. (42) and (B1), we obtain

$$
\begin{aligned}
& h=\frac{4 \omega_{*}^{2}}{9 \omega_{0}^{2}}+\frac{2 a^{2} \omega_{B i}^{2}}{9 \omega_{*}^{2}}, 2 \theta=-\xi \operatorname{sig}\left(k z_{0}\right) \frac{20}{9} a \frac{\omega_{B i}}{\omega_{0}}, \\
& z=\frac{\omega_{0} \tau}{2},
\end{aligned}
$$

which allows us to use the results of Appendix $\ddot{B}$. Using Eqs. (B12), (B9), (B11), and (43) we obtain the following expressions for the growth rate and the frequency of MHD oscillations:

$$
\begin{aligned}
& \frac{\gamma}{\omega_{*}}=\frac{225}{64 \sqrt{2}}\left(\frac{a \omega_{0} \omega_{B i}}{\omega_{*}^{2}}\right)^{2}\left(\frac{\omega_{0}}{\omega_{*}}\right)^{3}, \\
& \omega=\omega_{*}+\frac{7 a^{2} \omega_{0}^{2} \omega_{B i}^{2}}{16 \omega_{*}^{3}} \equiv \omega_{*}+\Delta \omega .
\end{aligned}
$$


The polarization vector of MHD oscillations with $\mathbf{k} \| \mathbf{B}_{0}$ in the pumping wave field $\mathbf{E}_{0} \| \mathbf{B}_{0}$ is obtained by substituting $\Delta \omega$ from Eq. (44) into Eq. (35).

In this and previous sections, we considered the excitation of MHD waves by the dipole pumping, but, naturally, with respect to certain types of oscillations, MHD waves themselves can play the role of pumping. The following section will consider one of such cases.

\section{EXCITATION OF WHISTLER OSCILLATIONS, PROPAGATING ACROSS THE EXTERNAL MAGNETIC FIELD, BY THE PUMPING FIELD $E_{0} \perp B_{0}$}

Let us consider the excitation of whistler oscillations with the wave vector $\mathbf{k}=(k, 0,0)$ by the pumping wave $\left.\mathbf{E}_{0}\right\rfloor \mathbf{B}_{0}$ (e.g., $A$ mode). Using Eq. (17), the expression for current $J_{k}(\omega, \mathbf{k})$ assumes the form [see Eq. (A5)]

$$
\begin{aligned}
J_{k}(\omega, \mathbf{k})= & -\frac{a \omega_{0}}{2} \frac{\partial \sigma_{k e}^{(i)}}{\partial \omega}\left(E_{e}^{+1} e^{i \theta}+E_{e}^{-1} e^{-i \theta}\right)-\frac{a \omega_{0}^{2}}{4} \frac{\partial^{2} \sigma_{k e}^{(i)}}{\partial \omega^{2}}\left\langle E_{e}^{+1} e^{i \theta}-E_{e}^{-1} e^{-i \theta}-\frac{a}{2}\left[E_{e}^{+2} e^{i 2 \theta}+E_{e}^{-2} e^{-i 2 \theta}+2 E_{e}(\omega)\right]\right\rangle \\
& -\frac{k V_{1}}{2}\left\langle\sigma_{k e}^{(i)}\left(\frac{E_{e}^{+1}}{\omega+\omega_{0}}-\frac{E_{e}^{-1}}{\omega-\omega_{0}}\right)-\frac{a \omega_{0}}{2} \frac{\partial \sigma_{k e}^{(i)}}{\partial \omega}\left(e^{i \theta} \frac{E_{e}^{+2}}{\omega+2 \omega_{0}}-e^{-i \theta} \frac{E_{e}^{-2}}{\omega-2 \omega_{0}}+\frac{E_{e}(\omega)}{\omega}\left(e^{-i \theta}-e^{i \theta}\right)\right)\right\rangle \\
& +\frac{k V_{e}}{2}\left\langle\sigma_{k 1}^{(i)}\left(\frac{E_{e}^{+1}}{\omega+\omega_{0}}+G^{e} \frac{E_{e}^{-1}}{\omega-\omega_{0}}\right)-\frac{a \omega_{0}}{2} \frac{\partial \sigma_{k 1}^{(i)}}{\partial \omega}\left(e^{i \theta} \frac{E_{e}^{+2}}{\omega+2 \omega_{0}}+G^{e} e^{-i \theta} \frac{E_{e}^{-2}}{\omega-2 \omega_{0}}+\frac{E_{e}(\omega)}{\omega}\left(e^{-i \theta}+G^{e} e^{i \theta}\right)\right)\right\rangle \\
& +\frac{V_{k}}{2}\left\langle\frac{k \sigma_{l e}^{(i)}}{\omega}\left(E_{e}^{+1}+G^{k} E_{e}^{-1}\right)+\omega_{0} \frac{\partial}{\partial \omega}\left(\frac{k \sigma_{l e}^{(i)}}{\omega}\right)\left(E_{e}^{+1}-G^{k} E_{e}^{-1}\right)-\frac{a \omega_{0}}{2} \frac{\partial}{\partial \omega}\left(\frac{k \sigma_{l e}^{(i)}}{\omega}\right)\left[e^{i \theta} E_{e}^{+2}+G^{k} e^{-i \theta} E_{e}^{-2}\right.\right. \\
& \left.+\left(e^{-i \theta}+G^{k} e^{i \theta}\right) E_{e}(\omega)\right]-\frac{k V_{1}}{2}\left[\frac{k \sigma_{l e}^{(i)}}{\omega}\left(\frac{E_{e}^{+2}}{\omega+2 \omega_{0}}-G^{k} \frac{E_{e}^{-2}}{\omega-2 \omega_{0}}\right)+\frac{k \sigma_{l e}^{(i)} E_{e}(\omega)}{\omega^{2}}\left(-1+G^{k}\right)\right] \\
& \left.+\frac{k V_{e}}{2}\left[\frac{k \sigma_{11}^{(i)}}{\omega}\left(\frac{E_{e}^{+2}}{\omega+2 \omega_{0}}+G^{e} G^{k} \frac{E_{e}^{-2}}{\omega-2 \omega_{0}}\right)+\frac{k \sigma_{11}^{(i)} E_{e}(\omega)}{\omega^{2}}\left(G^{e}+G^{k}\right)\right]\right) .
\end{aligned}
$$

Using Eq. (19), as in previous paragraphs, we obtain the dispersive equation (assuming $u_{1}^{2}=u_{2}^{2}$ ):

$$
\begin{aligned}
& \Lambda(\omega) E(\omega)+\frac{k u_{1}}{2 \omega}\left\langle\operatorname{sig}\left(k x_{0}\right) e_{m}^{*} \frac{\partial}{\partial \omega}\left(\omega \chi_{m n}^{(i)}\right) e_{n}\right. \\
& \left.-\operatorname{sig}\left(k x_{0}\right)\left(\chi_{11}^{(i)} e_{1}^{2}-\chi_{22}^{(i)} \widetilde{e}_{2}^{2}\right)\right\rangle\left(e^{i \theta} E^{+1}+e^{-i \theta} E^{-1}\right) \\
& +\frac{\left(k u_{1}\right)^{2}}{2 \omega^{2}}\left\langle\frac{1}{2} e_{m}^{*} \frac{\partial}{\partial \omega}\left(\omega^{2} \frac{\partial \chi_{m n}^{(i)}}{\partial \omega}\right) e_{n}+\chi_{11}^{(i)}\right. \\
& \left.-e_{1}^{2} \frac{\partial}{\partial \omega}\left(\omega \chi_{11}^{(i)}\right)+\vec{e}_{2}^{2} \frac{\partial}{\partial \omega}\left(\omega \chi_{22}^{(i)}\right)\right\rangle E(\omega)=0 .
\end{aligned}
$$

While obtaining Eq. (46) we, as before, neglected the terms proportional to $\omega_{0} / \omega$ and $\left[\left(a \omega_{0}\right)^{2} / \omega^{2}\right]\left(E^{+2} \pm E^{-2}\right)$, and instead used the relationships [see Eqs. (6) and (7)]

$$
a \omega_{0}=-k u_{1} \operatorname{sig}\left(k x_{0}\right), \quad \theta=(\pi / 2) \operatorname{sig}\left(k x_{0}\right)
$$

and introduced the notation

$$
\Lambda(\omega)=1+e_{i}^{*} \chi_{i j} e_{j}-\left(c^{2} k^{2} / \omega^{2}\right) \vec{e}_{2}^{2}
$$

Expanding into the series with respect to the undisturbed solution, Eq. (46) assumes the form

$$
\begin{aligned}
& \left\langle[\Lambda]_{*}^{\prime}\left(\omega-\omega_{*}\right)+\frac{1}{2}[\Lambda]_{*}^{\prime \prime}\left(\omega-\omega_{*}\right)^{2}\right\rangle E(\omega)-\frac{k u_{1}}{2}\left(e^{i \theta} E^{+1}+e^{-i \theta} E^{-1}\right) \operatorname{sig}\left(k x_{0}\right)\left\langle\left(\frac{1}{\omega}\left(\chi_{11}^{(i)} e_{1}^{2}-\chi_{22}^{(i)} e_{2}^{2}\right)\right.\right. \\
& \left.\left.-\frac{1}{\omega} e_{m}^{*} \frac{\partial}{\partial \omega}\left(\omega \chi_{m n}^{(i)}\right) e_{n}\right)_{*}+\left(\frac{1}{\omega}\left(\chi_{11}^{(i)} e_{1}^{2}-\chi_{22}^{(i)} \vec{e}_{2}^{2}\right)-\frac{1}{\omega} e_{m}^{*} \frac{\partial}{\partial \omega}\left(\omega \chi_{m n}^{(i)}\right) e_{n}\right)_{*}^{\prime}\left(\omega-\omega_{*}\right)\right\rangle \\
& +\frac{\left(k u_{1}\right)^{2}}{2 \omega_{*}^{2}}\left[\frac{1}{2} e_{m}^{*} \frac{\partial}{\partial \omega}\left(\omega^{2} \frac{\partial \chi_{m n}^{(i)}}{\partial \omega}\right) e_{n}+\chi_{11}^{(i)}-e_{1}^{2} \frac{\partial}{\partial \omega}\left(\omega \chi_{11}^{(i)}\right)+\vec{e}_{2}^{2} \frac{\partial}{\partial \omega}\left(\omega \chi_{22}^{(i)}\right)\right]_{*} E(\omega)=0
\end{aligned}
$$


Applying Eq. (39) to Eq. (47), after simple transformations we have

$$
\begin{gathered}
\frac{\partial^{2} E(\tau, \mathbf{k})}{\partial \tau^{2}}+\left(A+B \cos \omega_{0} \tau\right) \frac{\partial E(\tau, \mathbf{k})}{\partial \tau} \\
+\left(C+\mathscr{D} \cos \omega_{0} \tau\right) E(\tau, \mathbf{k})=0
\end{gathered}
$$

Writing Eq. (48) we have taken into account, as everywhere else, the smallness of $\omega_{0} / \omega_{*}$ and introduced the notation

$$
\begin{aligned}
A(\mathbf{k})= & -\left[2 i / \Lambda^{\prime \prime}\left(\omega_{*}, \mathbf{k}\right)\right]\left\langle\Lambda^{\prime}\left(\omega_{*}, \mathbf{k}\right)-\omega_{*} \Lambda^{\prime \prime}\left(\omega_{*}, \mathbf{k}\right)\right\rangle \\
B(\mathbf{k})= & \frac{2 i k u_{1}}{\Lambda^{\prime \prime}\left(\omega_{*}, \mathbf{k}\right)}\left(\frac{1}{\omega} e_{m}^{*} \frac{\partial}{\partial \omega}\left(\omega \chi_{m n}^{(i)}\right) e_{n}\right. \\
& \left.-\frac{1}{\omega}\left(e_{1}^{2} \chi_{11}^{(i)}-\widetilde{e}_{2}^{2} \chi_{22}^{(i)}\right)\right)_{*}^{\prime} \\
C(\mathbf{k})= & -\frac{2}{\Lambda^{\prime \prime}\left(\omega_{*}, \mathbf{k}\right)}\left\langle-\omega_{*} \Lambda^{\prime}\left(\omega_{*}, \mathbf{k}\right)+\frac{\omega_{*}^{2}}{2} \Lambda^{\prime \prime}\left(\omega_{*}, \mathbf{k}\right)\right. \\
& +\frac{\left(k u_{1}\right)^{2}}{2 \omega_{*}^{2}}\left[\frac{1}{2} e_{m}^{*} \frac{\partial}{\partial \omega}\left(\omega^{2} \frac{\partial \chi_{m n}^{(i)}}{\partial \omega}\right) e_{n}+\chi_{11}^{(i)}\right. \\
& \left.\left.-e_{1}^{2} \frac{\partial}{\partial \omega^{-}}\left(\omega \chi_{11}^{(i)}\right)+\vec{e}_{2}^{2} \frac{\partial}{\partial \omega}\left(\omega \chi_{22}^{(i)}\right)\right]_{*}\right\rangle \\
\mathscr{D}(\mathbf{k})= & \frac{2 k u_{1}}{\Lambda^{\prime \prime}\left(\omega_{*}, \mathbf{k}\right)}\left\langle\left(\frac{1}{\omega} e_{m}^{*} \frac{\partial}{\partial \omega}\left(\omega \chi_{m n}^{(i)}\right) e_{n}-\frac{1}{\omega}\left(e_{1}^{2} \chi_{11}^{(i)}\right.\right.\right. \\
& \left.\left.-\vec{e}_{2}^{2} \chi_{22}^{(i)}\right)\right)_{*}-\omega_{*}\left(\frac{1}{\omega} e_{m}^{*} \frac{\partial}{\partial \omega}\left(\omega \chi_{m n}^{(i)}\right) e_{n}\right. \\
& \left.\left.-\frac{1}{\omega}\left(e_{1}^{2} \chi_{11}^{(i)}-\vec{e}_{2}^{2} \chi_{22}^{(i)}\right)\right)_{*}^{\prime}\right\rangle
\end{aligned}
$$

Substituting Eq. (41) reduces Eq. (48) to the Mathieu equation:

$$
\begin{aligned}
& \frac{\partial^{2} \bar{E}(\tau)}{\partial \tau^{2}}+\left[C-\frac{1}{4}\left(A^{2}+\frac{B^{2}}{2}\right)\right. \\
& \left.+\left(\mathscr{D}-\frac{A B}{2}\right) \cos \omega_{0} \tau\right] \bar{E}(\tau)=0,
\end{aligned}
$$

where the terms $B \omega_{0} \sin \omega_{0} \tau$ and $B^{2} \cos 2 \omega_{0} \tau$ are neglected.

The solution of Eq. (22) for the whistler oscillations in question has the form ${ }^{9}$

$$
N^{2}=\left[\left(1+\chi_{1}\right)^{2}-\chi_{2}^{2}\right] /\left(1+\chi_{1}\right),
$$

where, for the case of a cold plasma,

$$
\begin{aligned}
& \chi_{1}=-\frac{\omega_{p i}^{2}}{\omega^{2}}\left(1+\frac{\omega_{B i}^{2}}{\omega^{2}}\right), \quad \chi_{2}=-\frac{\omega_{p i}^{2}}{\omega \omega_{B i}}\left(1+\frac{\omega_{B i}^{2}}{\omega^{2}}\right), \\
& \omega_{B i}^{2}<\omega^{2}<\omega_{B i}\left|\omega_{B e}\right| .
\end{aligned}
$$

In the dense plasma $\left(\omega_{p e}^{2}>\omega_{B e}^{2}\right)$, we obtain $\omega_{*}^{2}=k^{2} V_{A}^{2}$ from Eq. (50).
Using Eqs. (28), (29), (50), and (51), we can write expressions for coefficients in Eqs. (41) and (49). Omitting simple but fairly unwieldy calculations, we will have

$$
\begin{aligned}
& A=i_{3}^{2} \omega_{*}\left[4-3\left(\omega_{B i}^{2} / \omega^{2}\right)\right], \quad B=i 2 k u_{1}, \\
& C-\frac{1}{4}\left(A^{2}+\frac{B^{2}}{2}\right)=\frac{\omega_{*}^{2}}{9}\left(1-6 \frac{\omega_{B i}^{2}}{\omega_{*}^{2}}\right)+\frac{\left(k u_{1}\right)^{2} \omega_{B i}^{2}}{6 \omega_{*}^{2}} \\
& \mathscr{D}-\frac{A B}{2}=-\frac{2 k u_{1} \omega_{B i}^{2}}{3 \omega_{*}} .
\end{aligned}
$$

From comparing Eqs. (49) and (B1) we obtain

$$
\begin{aligned}
& h=\frac{4 \omega_{*}^{2}}{9 \omega_{0}^{2}}\left(1-6 \frac{\omega_{B i}^{2}}{\omega_{*}^{2}}\right)+\frac{2\left(k u_{1}\right)^{2} \omega_{B i}^{2}}{3 \omega_{0}^{2} \omega_{*}^{2}}, \\
& 2 \theta=\frac{8\left(k u_{1}\right) \omega_{B i}^{2}}{3 \omega_{*} \omega_{0}^{2}}, \quad z=\frac{\omega_{0} \tau}{2},
\end{aligned}
$$

which allows us to use the results of Appendix B. Using Eqs. (B12), (B9), (B11), and (52), we obtain the growth rate and frequency of whistler oscillations:

$$
\begin{aligned}
& \frac{\gamma}{\omega_{*}}=\frac{81}{16 \sqrt{2}}\left(\frac{k u_{1} \omega_{B i}^{2}}{\omega_{*}^{3}}\right)^{2}\left(\frac{\omega_{0}}{\omega_{*}}\right)^{3}, \\
& \omega=\omega_{*}-\frac{\left(k u_{1}\right)^{2} \omega_{B i}^{2}}{4 \omega_{*}^{3}} \equiv \omega_{*}+\Delta \omega .
\end{aligned}
$$

Substituting the obtained frequency value in Eq. (29) and expanding, we obtain the expression for the polarization vector:

$$
\begin{aligned}
\mathrm{e}= & \frac{1}{\left(1+\omega_{B i}^{2} / \omega_{*}^{2}\right)^{1 / 2}}\left\langle\left(1+\frac{\omega_{B i}^{2}|\Delta \omega|}{\omega_{*}^{3}}\right), i \frac{\omega_{B i}}{\omega_{*}}(1 .\right. \\
& \left.\left.-\frac{|\Delta \omega|}{\omega_{*}}\right)\right\rangle .
\end{aligned}
$$

It is seen from Eq. (54) that the pumping field tends to make the whistler oscillations quasilongitudinal.

The expression for the increment includes small parameter $\omega_{B i}^{2} / \omega_{*}^{2}$, therefore the obtained result is meaningful at a not very small relation $\omega_{B i}^{2} / \omega_{*}^{2}$. Substantially greater growth rate [of the Eq. (53) type, but without the small parameter $\left.\omega_{B i}^{2} / \omega_{*}^{2}\right]$ can be obtained considering the excitation of whistler oscillations, propagating at an angle $\cos ^{2} \theta \sim m_{e} / m_{i}\left(\cos \theta=k_{z} / k\right)$.

Let us note in conclusion, that, since we considered a cold collisionless plasma, $\delta=0$ [see Eq. (22)]. In the case $\delta \neq 0$, naturally, to determine the threshold value of the pumping wave amplitude it is necessary to compare the obtained increments with the linear decrement.

\section{CONCLUSION}

In the present paper, we have considered the excitation of electromagnetic oscillations in the field of the lowerfrequency dipole pumping. Without setting the aim of exhaustive study the dispersive equation (16), we have lim- 
ited ourselves to the consideration of three particular cases differing in the mutual orientation of vectors $\mathbf{k}, \mathbf{E}$, and $\mathbf{E}_{0}$. However, the proposed methods for the solution of the dispersive equation in the lower-frequency pumping field (naturally, within the framework of the employed restrictions) allow us to study the dispersive properties of arbitrary modes.

\section{ACKNOWLEDGMENTS}

This work was supported by NASA Grants No. NAGW-1619 and NAG5-1500 as well as NAF Grant No. ATM-9114409.

\section{APPENDIX A: OBTAINING EXPRESSION (15)}

Let us write Eq. (12b) in the following form:

$$
\begin{aligned}
j_{k}^{(i)}(\omega, \mathbf{k})= & \sum_{p, q} e^{i q \theta} J_{p} J_{p+q}\left\{\sigma _ { k m } ^ { ( i ) } ( \omega - p \omega _ { 0 } ) \left\langleE_{m}\left(\omega+q \omega_{0}\right)-\frac{V_{s} k_{s}}{2}\left(\frac{E_{m}\left[\omega+(q+1) \omega_{0}\right]}{\omega+(q+1) \omega_{0}}+G^{s} \frac{E_{m}\left[\omega+(q-1) \omega_{0}\right]}{\omega+(q-1) \omega_{0}}\right)\right.\right. \\
& \left.+\frac{V_{n} k_{m}}{2}\left(\frac{E_{n}\left[\omega+(q+1) \omega_{0}\right]}{\omega+(q+1) \omega_{0}}+G^{n} \frac{E_{n}\left[\omega+(q-1) \omega_{0}\right]}{\omega+(q-1) \omega_{0}}\right)\right\rangle+\frac{V_{k}}{2}\left[\frac{k_{e} \sigma_{e m}^{(i)}\left[\omega-(p-1) \omega_{0}\right]}{\omega-(p-1) \omega_{0}}\right. \\
& \times\left\langle E_{m}\left[\omega+(q+1) \omega_{0}\right]-\frac{V_{s} k_{s}}{2}\left(\frac{E_{m}\left[\omega+(q+2) \omega_{0}\right]}{\omega+(q+2) \omega_{0}}+G^{s} \frac{E_{m}\left(\omega+q \omega_{0}\right)}{\omega+q \omega_{0}}\right)+\frac{V_{n} k_{m}}{2}\left(\frac{E_{n}\left[\omega+(q+2) \omega_{0}\right]}{\omega+(q+2) \omega_{0}}\right.\right. \\
& \left.\left.+G^{n} \frac{E_{n}\left(\omega+q \omega_{0}\right)}{\omega+q \omega_{0}}\right)\right\rangle+G^{k} \frac{k_{e} \sigma_{e m}^{(i)}\left[\omega-(p+1) \omega_{0}\right]}{\omega-(p+1) \omega_{0}}\left\langle E_{m}\left[\omega+(q-1) \omega_{0}\right]-\frac{V_{s} k_{s}}{2}\left(\frac{E_{m}\left(\omega+q \omega_{0}\right)}{\omega+q \omega_{0}}\right.\right. \\
& \left.\left.\left.+G^{s} \frac{E_{m}\left[\omega+(q-2) \omega_{0}\right]}{\omega+(q-2) \omega_{0}}\right)+\frac{V_{n} k_{m}}{2}\left(\frac{E_{n}\left(\omega+q \omega_{0}\right)}{\omega+q \omega_{0}}+G^{n} \frac{E_{n}\left[\omega+(q-2) \omega_{0}\right]}{\omega+(q-2) \omega_{0}}\right)\right\rangle\right\} .
\end{aligned}
$$

In the written expression for the current, let us expand the electric fields into the Taylor series with respect to point $(\omega, \mathbf{k})$, then after summing up over $q$, Eq. (A1) assumes the form

$$
\begin{aligned}
j_{k}^{(i)}(\omega, \mathbf{k})= & \sum_{p} J_{p}\left\{\sigma _ { k m } ^ { ( i ) } ( \omega - p \omega _ { 0 } ) \sum _ { r = 0 } ^ { \infty } \frac { 1 } { r } ( \frac { \omega _ { 0 } } { i } ) ^ { r } \frac { \partial ^ { r } } { \partial \omega ^ { r } } \left\langleE_{m}(\omega) \frac{\partial^{r}}{\partial \theta^{r}}-\frac{V_{s} k_{s}}{2}\left(\frac{E_{m}(\omega)}{\omega}\right)\left(e^{-i \theta} \frac{\partial^{r}}{\partial \theta^{r}} e^{i \theta}+G^{s} e^{i \theta} \frac{\partial^{r}}{\partial \theta^{r}} e^{-i \theta}\right)\right.\right. \\
& \left.+\frac{V_{n} k_{m}}{2}\left(\frac{E_{n}(\omega)}{\omega}\right)\left(e^{-i \theta} \frac{\partial^{r}}{\partial \theta^{r}} e^{i \theta}+G^{n} e^{i \theta} \frac{\partial^{r}}{\partial \theta^{r}} e^{-i \theta}\right)\right\rangle \\
& +\frac{V_{k}}{2}\left[\frac { k _ { e } \sigma _ { e m } ^ { ( i ) } [ \omega - ( p - 1 ) \omega _ { 0 } ] } { \omega - ( p - 1 ) \omega _ { 0 } } \sum _ { r = 0 } ^ { \infty } \frac { 1 } { r ! } ( \frac { \omega _ { 0 } } { i } ) ^ { r } \frac { \partial ^ { r } } { \partial \omega ^ { r } } \left\langleE_{m}(\omega) e^{-i \theta} \frac{\partial^{r}}{\partial \theta^{r}} e^{i \theta}-\frac{V_{s} k_{s}}{2}\left(\frac{E_{m}(\omega)}{\omega}\right)\left(e^{-i 2 \theta} \frac{\partial^{r}}{\partial \theta^{r}} e^{i 2 \theta}\right.\right.\right. \\
& \left.\left.+G^{s} \frac{\partial^{r}}{\partial \theta^{r}}\right)+\frac{V_{n} k_{m}}{2}\left(\frac{E_{n}(\omega)}{\omega}\right)\left(e^{-i 2 \theta} \frac{\partial^{r}}{\partial \theta^{r}} e^{i 2 \theta}+G^{n} \frac{\partial^{r}}{\partial \theta^{r}}\right)\right\rangle \\
& +G^{k} \frac{k_{e} \sigma_{e m}^{(i)}\left[\omega-(p+1) \omega_{0}\right]}{\omega-(p+1) \omega_{0}} \sum_{r=0}^{\infty} \frac{1}{r !}\left(\frac{\omega_{0}}{i}\right)^{r} \frac{\partial^{r}}{\partial \omega^{r}}\left\langle E_{m}(\omega) e^{i \theta} \frac{\partial^{r}}{\partial \theta^{r}} e^{-i \theta}-\frac{V_{s} k_{s}}{2}\left(\frac{E_{m}(\omega)}{\omega}\right)\left(\frac{\partial^{r}}{\partial \theta^{r}}\right.\right. \\
& \left.\left.\left.+G^{s} e^{i 2 \theta} \frac{\partial^{r}}{\partial \theta^{r}} e^{-i 2 \theta}\right)+\frac{V_{n} k_{m}}{2}\left(\frac{E_{n}(\omega)}{\omega}\right)\left(\frac{\partial^{r}}{\partial \theta^{r}}+G^{n} e^{i 2 \theta} \frac{\partial^{r}}{\partial \theta^{r}} e^{-i 2 \theta}\right)\right\rangle\right] e^{i \theta \sin \theta-i p \theta} .
\end{aligned}
$$

Writing Eq. (A2), we used obvious relationships:

$$
\left(\omega_{0} q\right)^{r} e^{i q \theta}=\left(\frac{\omega_{0}}{i}\right)^{r} \frac{\partial^{r}}{\partial \theta^{r}} e^{i q \theta} \quad(r=0,1,2, \ldots)
$$

and

$$
\sum_{q=-\infty}^{\infty} J_{q}(a) e^{ \pm i q \theta}=e^{ \pm i a \sin \theta}
$$

Similarly, we can expand the conductivity into the Taylor series and sum over $p$. Then 


$$
\begin{aligned}
j_{k}^{(i)}(\omega, \mathbf{k})= & \sum_{r, t=0}^{\infty} \frac{1}{r ! t !}\left(\frac{\omega_{0}}{i}\right)^{r+t}\left\{\frac { \partial ^ { r } } { \partial \omega ^ { r } } \left\langleE_{m}(\omega) \frac{\partial^{r}}{\partial \theta^{r}}-\frac{V_{s} k_{s}}{2}\left(\frac{E_{m}(\omega)}{\omega}\right)\left(e^{-i \theta} \frac{\partial^{r}}{\partial \theta^{r}} e^{i \theta}+G^{s} e^{i \theta} \frac{\partial^{r}}{\partial \theta^{r}} e^{-i \theta}\right)+\frac{V_{n} k_{m}}{2}\left(\frac{E_{n}(\omega)}{\omega}\right)\right.\right. \\
& \left.\times\left(e^{-i \theta} \frac{\partial^{r}}{\partial \theta^{r}} e^{i \theta}+G^{n} e^{i \theta} \frac{\partial^{r}}{\partial \theta^{r}} e^{-i \theta}\right)\right\rangle \frac{\partial^{t}}{\partial \omega^{t}} \sigma_{k m}^{(i)}(\omega) e^{i a \sin \theta} \frac{\partial^{t}}{\partial \theta^{t}} e^{-i \theta \sin \theta}+\frac{V_{k}}{2}\left[\frac { \partial ^ { r } } { \partial \omega ^ { r } } \left\langleE_{m}(\omega) e^{-i \theta} \frac{\partial^{r}}{\partial \theta^{r}} e^{i \theta}\right.\right. \\
& -\frac{V_{s} k_{s}}{2}\left(\frac{E_{m}(\omega)}{\omega}\right)\left(e^{-i 2 \theta} \frac{\partial^{r}}{\partial \theta^{r}} e^{i 2 \theta}+G^{s} \frac{\partial^{r}}{\partial \theta^{r}}\right)+\frac{V_{n} k_{m}}{2}\left(\frac{E_{n}(\omega)}{\omega}\right)\left(e^{-i 2 \theta} \frac{\partial^{r}}{\partial \theta^{r}} e^{i 2 \theta}\right. \\
& \left.\left.+G^{n} \frac{\partial^{r}}{\partial \theta^{r}}\right)\right\rangle \frac{\partial^{t}}{\partial \omega^{t}}\left(\frac{k_{e} \sigma_{e m}^{(i)}(\omega)}{\omega}\right) e^{i a \sin \theta-i \theta} \frac{\partial^{t}}{\partial \theta^{t}} e^{-i a \sin \theta+i \theta}+G^{k} \frac{\partial^{r}}{\partial \omega^{r}}\left\langle E_{m}(\omega) e^{i \theta} \frac{\partial^{r}}{\partial \theta^{r}} e^{-i \theta}-\frac{V_{s} k_{s}}{2}\left(\frac{E_{m}(\omega)}{\omega}\right)\right. \\
& \times\left(\frac{\partial^{r}}{\partial \theta^{r}}+G^{s} e^{i 2 \theta} \frac{\partial^{r}}{\partial \theta^{r}} e^{-i 2 \theta}\right)+\frac{V_{n} k_{m}}{2}\left(\frac{E_{n}(\omega)}{\omega}\right)\left(\frac{\partial^{r}}{\partial \theta^{r}}\right. \\
& \left.\left.\left.\left.+G^{n} e^{i 2 \theta} \frac{\partial^{r}}{\partial \theta^{r}} e^{-i 2 \theta}\right)\right\rangle \frac{\partial^{t}}{\partial \omega^{t}}\left(\frac{k_{e} \sigma_{e m}^{(i)}(\omega)}{\omega}\right) e^{i \alpha \sin \theta+i \theta} \frac{\partial^{t}}{\partial \theta^{t}} e^{-i \alpha \sin \theta-i \theta}\right]\right\} .
\end{aligned}
$$

We are interested in the case of the lower-frequency pumping, i.e., $\omega_{0}<\omega$, and besides, to rule out the possible flux instability, we will require the fulfillment of $k u_{0} \alpha<\omega$ ( $\alpha$ is determined by conductivity and the type of oscillations under consideration). These assumptions allow us to restrict ourselves to terms $t=0,1$, and 2 in the expansion of the linear conductivity and write

$$
\begin{aligned}
j_{k}^{(i)}(\omega, \mathbf{k})= & \sum_{r=0}^{\infty} \frac{1}{r !}\left(\frac{\omega_{0}}{i}\right)^{r}\left\{\frac { \partial ^ { r } } { \partial \omega ^ { r } } \left\langleE_{m}(\omega) \frac{\partial^{r}}{\partial \theta^{r}}-\frac{V_{s} k_{s}}{2}\left(\frac{E_{m}(\omega)}{\omega}\right)\left(e^{-i \theta} \frac{\partial^{r}}{\partial \theta^{r}} e^{i \theta}+G^{s} e^{i \theta} \frac{\partial^{r}}{\partial \theta^{r}} e^{-i \theta}\right)+\frac{V_{n} k_{m}}{2}\left(\frac{E_{n}(\omega)}{\omega}\right)\right.\right. \\
& \left.\times\left(e^{-i \theta} \frac{\partial^{r}}{\partial \theta^{r}} e^{i \theta}+G^{n} e^{i \theta} \frac{\partial^{r}}{\partial \theta^{r}} e^{-i \theta}\right)\right\rangle\left\langle\sigma_{k m}^{(i)}(\omega)-a \omega_{0} \cos \theta \frac{\partial \sigma_{k m}^{(i)}}{\partial \omega}-\frac{\omega_{0}^{2}}{2}\left(i a \sin \theta-a^{2} \cos ^{2} \theta\right) \frac{\partial^{2} \sigma_{k m}^{(i)}}{\partial \omega^{2}}\right\rangle \\
& +\frac{V_{k}}{2}\left[\frac { \partial ^ { r } } { \partial \omega ^ { r } } \left\langleE_{m}(\omega) e^{-i \theta} \frac{\partial^{r}}{\partial \theta^{r}} e^{i \theta}-\frac{V_{s} k_{s}}{2}\left(\frac{E_{m}(\omega)}{\omega}\right)\left(e^{-i 2 \theta} \frac{\partial^{r}}{\partial \theta^{r}} e^{i 2 \theta}+G^{s} \frac{\partial^{r}}{\partial \theta^{r}}\right)+\frac{V_{n} k_{m}}{2}\left(\frac{E_{n}(\omega)}{\omega}\right)\right.\right. \\
& \left.\times\left(e^{-i 2 \theta} \frac{\partial^{r}}{\partial \theta^{r}} e^{i 2 \theta}+G^{n} \frac{\partial^{r}}{\partial \theta^{r}}\right)\right\rangle\left\langle\frac{k_{e} \sigma_{e m}^{(i)}(\omega)}{\omega}+\omega_{0}(1-a \cos \theta) \frac{\partial}{\partial \omega}\left(\frac{k_{e} \sigma_{e m}^{(i)}(\omega)}{\omega}\right)\right\rangle \\
& +G^{k} \frac{\partial^{r}}{\partial \omega^{r}}\left\langle E_{m}(\omega) e^{i \theta} \frac{\partial^{r}}{\partial \theta^{r}} e^{-i \theta}-\frac{V_{s} k_{s}}{2}\left(\frac{E_{m}(\omega)}{\omega}\right)\left(\frac{\partial^{r}}{\partial \theta^{r}}+G^{s} e^{i 2 \theta} \frac{\partial^{r}}{\partial \theta^{r}} e^{-i 2 \theta}\right)+\frac{V_{n} k_{m}}{2}\left(\frac{E_{n}(\omega)}{\omega}\right)\left(\frac{\partial^{r}}{\partial \theta^{r}}\right.\right. \\
& \left.\left.\left.\left.+G^{n} e^{i 2 \theta} \frac{\partial^{r}}{\partial \theta^{r}} e^{-i 2 \theta}\right)\right\rangle\left\langle\frac{k_{e} \sigma_{e m}^{(i)}(\omega)}{\omega}-\omega_{0}(1+a \cos \theta) \frac{\partial}{\partial \omega}\left(\frac{k_{e} \sigma_{e m}^{(i)}(\omega)}{\omega}\right)\right\rangle\right]\right\} .
\end{aligned}
$$

Retaining in Eq. (A4) the terms proportional to $1, k u_{0}, k u_{0} \omega_{0},\left(k u_{0}\right)^{2}$, and summing over $r$, we finally obtain

$$
\begin{aligned}
j_{k}^{(i)}(\omega, \mathbf{k})= & \sigma_{k e}^{(i)}(\omega) E_{e}(\omega)-\frac{a \omega_{0}}{2} \frac{\partial}{\partial \omega}\left[\sigma_{k e}^{(i)}(\omega)\right]\left[e^{i \theta} E_{e}\left(\omega+\omega_{0}\right)+e^{-i \theta} E_{e}\left(\omega-\omega_{0}\right)\right]-\frac{a \omega_{0}^{2}}{4} \frac{\partial^{2}}{\partial \omega^{2}}\left[\sigma_{k e}^{(i)}(\omega)\right]\left\langle e^{i \theta} E_{e}\left(\omega+\omega_{0}\right)\right. \\
& \left.-e^{-i \theta} E_{e}\left(\omega-\omega_{0}\right)+\frac{a}{2}\left[e^{i 2 \theta} E_{e}\left(\omega+2 \omega_{0}\right)+e^{-i 2 \theta} E_{e}\left(\omega-2 \omega_{0}\right)+2 E_{e}(\omega)\right]\right)-\frac{k_{m} V_{n}}{2}\left\langle\sigma_{k e}^{(i)}(\omega)\right. \\
& \times\left(\delta_{n m} \frac{E_{e}\left(\omega+\omega_{0}\right)}{\omega+\omega_{0}}+G_{n m} \frac{E_{e}\left(\omega-\omega_{0}\right)}{\omega-\omega_{0}}\right)-\frac{a \omega_{0}}{2} \frac{\partial}{\partial \omega}\left[\sigma_{k e}^{(i)}(\omega)\right]\left(\delta_{n m} e^{i \theta} \frac{E_{e}\left(\omega+2 \omega_{0}\right)}{\omega+2 \omega_{0}}+G_{n m} e^{-i \theta} \frac{E_{e}\left(\omega-2 \omega_{0}\right)}{\omega-2 \omega_{0}}\right. \\
& \left.\left.+\frac{E_{e}(\omega)}{\omega}\left(\delta_{n m} e^{-i \theta}+G_{n m} e^{i \theta}\right)\right)\right\rangle+\frac{k_{e} V_{n}}{2}\left\langle\sigma_{k e}^{(i)}(\omega)\left(\delta_{n m} \frac{E_{m}\left(\omega+\omega_{0}\right)}{\omega+\omega_{0}}+G_{n m} \frac{E_{m}\left(\omega-\omega_{0}\right)}{\omega-\omega_{0}}\right)\right. \\
& \left.-\frac{a \omega_{0}}{2} \frac{\partial}{\partial \omega}\left[\sigma_{k e}^{(i)}(\omega)\right]\left(\delta_{n m} e^{i \theta} \frac{E_{m}\left(\omega+2 \omega_{0}\right)}{\omega+2 \omega_{0}}+G_{n m} e^{-i \theta} \frac{E_{m}\left(\omega-2 \omega_{0}\right)}{\omega-2 \omega_{0}}+\frac{E_{m}(\omega)}{\omega}\left(\delta_{n m} e^{-i \theta}+G_{n m} e^{i \theta}\right)\right)\right\rangle \\
& +\frac{V_{n}}{2}\left[\frac{k_{m} \sigma_{m e}^{(i)}(\omega)}{\omega}\left[\delta_{n k} E_{e}\left(\omega+\omega_{0}\right)+G_{n k} E_{e}\left(\omega-\omega_{0}\right)\right]+\omega_{0} \frac{\partial}{\partial \omega}\left(\frac{k_{m} \sigma_{m e}^{(i)}(\omega)}{\omega}\right)\right.
\end{aligned}
$$




$$
\begin{aligned}
& \times\left[\delta_{n k} E_{e}\left(\omega+\omega_{0}\right)-G_{n k} E_{e}\left(\omega-\omega_{0}\right)\right]-\frac{a \omega_{0}}{2} \frac{\partial}{\partial \omega}\left(\frac{k_{m} \sigma_{m e}^{(i)}(\omega)}{\omega}\right)\left[\delta_{n k} e^{i \theta} E_{e}\left(\omega+2 \omega_{0}\right)+G_{n k} e^{-i \theta} E_{e}\left(\omega-2 \omega_{0}\right)\right. \\
& \left.+E_{e}(\omega)\left(\delta_{n k} e^{-i \theta}+G_{n k} e^{i \theta}\right)\right]-\frac{k_{p} V_{q}}{2}\left\langle\frac{k_{m} \sigma_{m e}^{(i)}(\omega)}{\omega}\left(\delta_{q p} \delta_{n k} \frac{E_{e}\left(\omega+2 \omega_{0}\right)}{\omega+2 \omega_{0}}+G_{q p} G_{n k} \frac{E_{e}\left(\omega-2 \omega_{0}\right)}{\omega-2 \omega_{0}}\right)\right. \\
& \left.+\frac{k_{m} \sigma_{m e}^{(i)}(\omega) E_{e}(\omega)}{\omega^{2}}\left(G_{q p} \delta_{n k}+\delta_{q p} G_{n k}\right)\right\rangle+\frac{k_{e} V_{p}}{2}\left(\frac{k_{m} \sigma_{m e}^{(i)}(\omega)}{\omega}\left(\delta_{q p} \delta_{n k} \frac{E_{q}\left(\omega+2 \omega_{0}\right)}{\omega+2 \omega_{0}}+G_{q p} G_{n k} \frac{E_{q}\left(\omega-2 \omega_{0}\right)}{\omega-2 \omega_{0}}\right)\right. \\
& \left.\left.+\frac{k_{m} \sigma_{m e}^{(i)}(\omega) E_{q}(\omega)}{\omega^{2}}\left(G_{q p} \delta_{n k}+\delta_{q p} G_{n k}\right)\right\rangle\right]
\end{aligned}
$$

Writing Eq. (A5), unlike Eq. (12), we used a mathematically more accurate writing, introducing the tensor

$$
G_{n k}=\left(\begin{array}{ccc}
-1 & 0 & 0 \\
0 & 1 & 0 \\
0 & 0 & -1
\end{array}\right)
$$

Finally, we can obtain Eq. (A5) in an abridged form as well:

$$
j_{k}^{(i)}(\omega, \mathbf{k})=\sigma_{k e}^{(i)}(\omega, \mathbf{k}) E_{e}(\omega, \mathbf{k})+J_{k}(\omega, \mathbf{k})
$$

\section{APPENDIX B: THE SOLUTION OF THE MATHIEU EQUATION AT GREAT $h$ AND $|\theta|$}

We shall consider

$$
\frac{\partial^{2} g(z, \mathbf{k})}{\partial z^{2}}+(h-2 \theta \cos 2 z) g(z, \mathbf{k})=0
$$

as a standard form of the Mathieu equation. ${ }^{10}$

It is known from the theory of the Mathieu equation that, if the solution of Eq. (B1) satisfies boundary conditions

$$
g(0)=1, \quad \frac{\partial g(0)}{\partial z}=0
$$

then characteristic index $v$ is connected with the solution in the following way:

$$
\operatorname{ch}(v \pi)=g(\pi) \text {. }
$$

In the present paper we assume that $\omega_{0}<\omega_{*}$ therefore $h>1$. Moreover, the correctness of the expansion used [see
Eq. (A5)] requires the fulfillment of $h>2|\theta|$. Apart from these obvious inequalities we shall require that $2|\theta|>1$. While fulfilling these inequalities, we can use the Loisville transformation ${ }^{10}$ to solve (B1):

$$
x=\int_{0}^{z}\left(1-\frac{2 \theta}{h} \cos 2 t\right)^{1 / 2} d t ; \quad \eta=(h-2 \theta \cos 2 z)^{1 / 4} g .
$$

Then Eq. (B1) assumes the form

$$
\frac{\partial^{2} \eta}{\partial x^{2}}+h[1+r(x)] \eta=0
$$

where

$$
r(x)=\frac{\theta^{2}\left(4+\sin ^{2} 2 z\right)-2 \theta h \cos 2 z}{(h-2 \theta \cos 2 z)^{3}}
$$

Taking into account the fact that $h>1$ and $h>2|\theta|$, let us rewrite $r(x)$ to an accuracy of up to terms of order $\epsilon^{2}$ (where $\epsilon=2 \theta / h$ ):

$$
h r(x)=-\epsilon \cos 2 x-\left(\epsilon^{2} / 8\right)(1+15 \cos 4 x) .
$$

Equation (B3) is solved by the method of successive approximations over parameter $\epsilon\left(\eta=\eta_{0}+\eta_{1}+\eta_{2}\right)$ :

(0): $\eta_{0}=(h-2 \theta)^{1 / 4} \cos \left[h-\left(\epsilon^{2} / 8\right)\right]^{1 / 2} x$,

(1): $\quad \eta_{1}=(h-2 \theta)^{1 / 4} \frac{\epsilon}{8}\left(\frac{2 \cos h^{1 / 2} x}{1-h}-\frac{\cos \left(h^{1 / 2}+2\right) x}{1+h^{1 / 2}}\right.$

$$
\left.-\frac{\cos \left(h^{1 / 2}-2\right) x}{1-h^{1 / 2}}\right)
$$

(2): $\quad \eta_{2}=(h-2 \theta)^{1 / 4} \frac{\epsilon^{2}}{16}\left[\left(\frac{1}{(1-h)^{2}}+\frac{15}{2(4-h)}-\frac{2+h}{4(1-h)(4-h)}\right) \cosh ^{1 / 2} x-\frac{1}{2(1-h)}\left(\frac{\cos \left(h^{1 / 2}+2\right) x}{1+h^{1 / 2}}+\frac{\cos \left(h^{1 / 2}-2\right) x}{1-h^{1 / 2}}\right)\right.$

$$
\left.-\frac{\cos \left(h^{1 / 2}+4\right) x}{8\left(2+h^{1 / 2}\right)}\left(15-\frac{1}{1+h^{1 / 2}}\right)-\frac{\cos \left(h^{1 / 2}-4\right) x}{8\left(2-h^{1 / 2}\right)}\left(15-\frac{1}{1-h^{1 / 2}}\right)-\frac{x}{(1-h) h^{1 / 2}} \sinh ^{1 / 2} x\right] .
$$


When writing Eqs. (B4)-(B6), we employed boundary conditions.

Let us substitute $x(\pi) \simeq \pi\left(1-\epsilon^{2} / 16\right)$ in the obtained solution of the Mathieu equation and, restricting ourselves to terms up to $\epsilon^{2}$ inclusive, reduce $g(\pi)$ to the form

$$
\begin{aligned}
g(\pi)= & \cosh ^{1 / 2} \pi\left(1-\frac{\epsilon^{2}}{16}-\frac{\epsilon^{2}}{16 h}\right) \\
& -\frac{\epsilon^{2} \pi}{16(1-h) h^{1 / 2}} \sinh ^{1 / 2} \pi\left(1-\frac{\epsilon^{2}}{16}\right) .
\end{aligned}
$$

Singling out the real and imaginary parts $v=i v^{\prime}+v^{\prime \prime}$ in the characteristic index, let us rewrite (B2):

$$
\operatorname{ch}\left(v^{\prime \prime} \pi\right) \cos \left(v^{\prime} \pi\right)+i s h\left(v^{\prime \prime} \pi\right) \sin \left(v^{\prime} \pi\right)=g(\pi),
$$

and, in order that $v^{\prime \prime} \neq 0$, it is necessary to require $v^{\prime}=n$ $(n=1,2, \ldots)$.

Substituting Eq. (B7) in Eq. (B8) and introducing the relative frequency shift

$$
\left(\nu^{\prime}\right)^{2}=h-\Delta=n^{2}, \quad h^{1 / 2} \approx n+\Delta / 2 n
$$

one can write

$$
\begin{aligned}
\operatorname{ch}\left(v^{\prime \prime} \pi\right)= & 1-\frac{\pi^{2}}{8}\left(\frac{\Delta}{n}-\frac{\epsilon^{2} n}{8}-\frac{\epsilon^{2}}{8 n}\right)^{2} \\
& -\frac{\epsilon^{2} \pi^{2}}{32(1-h) h^{1 / 2}}\left(\frac{\Delta}{n}-\frac{\epsilon^{2} n}{8}\right) .
\end{aligned}
$$

Expanding $\operatorname{ch}\left(v^{\prime \prime} \pi\right)$, we rewrite Eq. (B10a) in the form

$$
4 h\left(v^{\prime \prime}\right)^{2}=-\left(\Delta-\frac{\epsilon^{2} n}{8}-\frac{\epsilon^{2}}{8}\right)^{2}+\frac{\epsilon^{2}}{4 h}\left(\Delta-\frac{\epsilon^{2} h}{8}\right) .
$$

The increment's extremum is reached at frequency shift

$$
\Delta=\epsilon^{2} h / 8+\epsilon^{2} / 8
$$

and takes the value

$$
v_{\text {ext }}^{\prime \prime}=\frac{\epsilon^{2}}{8 \sqrt{2} h} \text {. }
$$

The mechanism of the studied instability can be understood from analyzing Eqs. (B4)-(B6). In the first order over $\epsilon$ [see Eq. (B5)], the interaction of the proper mode $\left(h^{1 / 2}, \mathbf{k}\right)$ of the undisturbed plasma with the pumping wave generates oscillations with spectrum $\left(h^{1 / 2} \pm 2, \mathbf{k}\right)$, whereas the interaction of these oscillations with the external field leads to the growth of the amplitude of the proper mode $\left(h^{1 / 2}, \mathbf{k}\right)$ [the last term in Eq. (B6)]. In other words, the beats of high-frequency waves $\left(h^{1 / 2}, \mathbf{k}\right)$ and $\left(h^{1 / 2} \pm 2, \mathbf{k}\right)$ are in resonance with the lower-frequency pumping wave.

${ }^{1}$ V. S. Krivitsky, Yu. M. Prydko, and V. N. Tsytovich, Fiz. Plasmy 16, 801 (1990).

${ }^{2}$ V. S. Krivitsky and V. N. Tsytovich, Conrid. Plasma Phys. 30, 339 (1990).

${ }^{3}$ S. N. Sarma, R. N. Khound, S. Bujarbarua, and M. Nambu, J. Phys. Soc. Jpn. 57, 3029 (1988).

${ }^{4}$ V. P. Silin, Parametric Effects of Powerful Radiation on Plasma (Nakka, Moscow, 1973).

${ }^{5}$ Electrodynamics of Plasma, edited by A. I. Akhiezer (Nauka, Moscow, 1974).

${ }^{6}$ Fundamenals of Plasma Physics, edited by A. A. Galeev and R. Sudan (Energoatomizdat, Moscow, 1984), Vol. 2.

${ }^{7}$ L. D. Landau and E. M. Lifshits, The Field Theory (Nauka, Moscow, 1988), Vol. 2.

${ }^{8} \mathrm{G}$. Korn and T. Korn, Mathematical Handbook (McGraw-Hill, New York, 1968).

${ }^{9}$ A. F. Alexandrov, L. S. Bogdankevich, and A. A. Rukhadze, Fundamentals of Plasma Electrodynamics (Vyshaya Shkola, Moscow, 1988).

${ }^{10} \mathrm{H}$. Bateman and A. Erdelyi, Higher Träscendental Functions. Elliptic and Automorphic Functions, Lame and Mathieu Functions (Nauka, Moscow, 1967). 